\title{
Impact of MRSA Transmission and Infection in a Neonatal Intensive Care Unit in China: A Bundle Intervention Study during 2014-2017
}

\author{
Huiping Huang $\mathbb{D},{ }^{1,2}$ Jing Ran, ${ }^{3}$ Jianzhou Yang $\mathbb{D}^{1},{ }^{1}$ Peng $L i \mathbb{D},{ }^{1}$ and Guihua Zhuang $\mathbb{D}^{1}$ \\ ${ }^{1}$ School of Public Health, Health Science Center, Xian Jiaotong University, 76 West Yanta Road, Xian 710061, China \\ ${ }^{2}$ Department of Infection Control, The First Affiliated Hospital of Xiamen University, 55 Zhenhai Road, Xiamen, 361003, China \\ ${ }^{3}$ Department of Gynecology and Obstetrics, The First Affiliated Hospital of Xiamen University, 55 Zhenhai Road, \\ Xiamen, 361003, China
}

Correspondence should be addressed to Guihua Zhuang; zhuanggh@mail.xjtu.edu.cn

Received 14 February 2019; Accepted 20 June 2019; Published 10 July 2019

Guest Editor: Yatao Liu

Copyright (C) 2019 Huiping Huang et al. This is an open access article distributed under the Creative Commons Attribution License, which permits unrestricted use, distribution, and reproduction in any medium, provided the original work is properly cited.

\begin{abstract}
Objective. To evaluate the efficacy of bundle intervention on healthcare-associated (HA) methicillin-resistant Staphylococcus Aureus (MRSA) infection in the neonatal intensive care unit (NICU). Methods. In this study, 11,277 infants having undergone treatment at the NICU in Xiamen, China, from January 2014 to February 2017 were recruited. We retrospectively reviewed patients' demographic and clinical information. Patients from 2014 to 2015 were treated as the control group and those from 2016 to 2017 were classified as the experimental group. Bundle intervention measures were performed, including screening for MRSA, isolation precautions, training of hand hygiene, cleaning protocols, and decontamination of isolation ward. The HA-MRSA data and compliance of infection control measures between both groups were analyzed. Results. Through bundle interventions, the compliance with the isolation of MRSA raised from $55.88 \%$ to $92.86 \%$ and hand hygiene compliance increased from $90.07 \%$ to $93.23 \%(P<0.05)$. The HA infection decreased from $1.87 \%$ to $1.71 \%(P>0.05)$ and HA detection rate of MRSA declined from $2.63 \%$ o to $1.00 \%$, respectively $(P<0.05)$. Conclusion. Multifaceted interventions can effectively prevent MRSA infection and transmission; this includes active surveillance, isolation precautions, increased hand hygiene compliance, environmental cleaning, and decontamination.
\end{abstract}

\section{Introduction}

Methicillin-resistant Staphylococcus aureus (MRSA) is one type of multidrug resistant organisms (MDROs) which can remain in the environment for a long term [1]. With the broad-spectrum antimicrobial widely used in clinical settings, incidences of MRSA infection are growing worldwide [2]. MRSA refers to a major healthcare-associated infection (HAI) organism associated with an increasing morbidity and mortality rate. As neonatal immunologic function is immature, MRSA infections occur more frequently in neonatal intensive care units (NICUs). MRSA is reported to be associated with the infection of skin and soft tissue (SSTI) as well as respiratory tracts. In the meantime, HA-MRSA is the main cause of pneumonia, osteomyelitis, and bacteremia $[3,4]$. Most neonatal bloodstream infections (BSI) are MRSArelated sepsis which are associated with a high mortality rate
[5-7]. HA-MRSA infections are more aggressive and difficult to diagnose and treat which leads to higher mortality rates, longer hospital stays, and increased financial burdens $[8,9]$.

The prevalence of MRSA is increasing in neonates [10,11]. MRSA can be spread from environment to patients, and it can also be transmitted by colonized or infected patients [1, 12]. Before a proper cleaning, medical devices (stethoscopes, otoscopes, and thermometers) and various objects in hospital environments were positive for Staphylococcus spp., which has been associated with transmission of HAI [1]. MRSA is subject to a higher risk of transmission than MSSA [13, 14]. Control of HA-MRSA remains challenging in NICUs: the recent research identified contaminated or dirty wound operations and MRSA colonization during hospitalization as risk factors for SSI in neonates [15]. Studies demonstrated MRSA had become endemic in numerous NICUs and caused invasive disease and, in some cases, even death [16-18]. For 
instance, the majority of outbreaks (in six out of 10) in North London were infected with MRSA [19]. Stricter adherence to disinfection practices by healthcare professionals would help to alleviate outbreak and transmission.

The published research demonstrated that the incidence of MRSA in hospital settings had decreased steadily since 2005 in the US, and this decline may be due to increased attention to infection prevention [20]. Several recent studies also have shown that HA-MRSA decreased significantly after the implementation of the intervention methods [21,22]. The Society for Healthcare Epidemiology of America (SHEA) has developed guidelines for the prevention of transmission of MRSA within healthcare settings; chief among the recommendations is to improve hand hygiene. Infection control measures were performed to prevent transmission and reduce the risk of acquiring HA-MRSA (e.g., isolation precautions, active surveillance, and hand hygiene compliance). However, whether these measures are effective or sufficient remains controversial [23-25]. Initiative through universal screening and isolation to prevent MRSA infections was considered cost effective [26]; however, universal surveillance results in an overall increase in costs [27]. A patient-centered care bundle intervention is effective but not cost efficient. MRSA are often transmitted through contact with healthcare personnel. The success of an infection control program requires bundle intervention, effective leadership, and a positive work environment. To achieve success, it also relies heavily on the cooperation and participation of healthcare personnel in both behavioral and practice changes [28].

The research studies of the bundle interventions for MRSA are limited, especially in developing countries [17]. The preventability of HA-MRSA in NICU's has rarely been evaluated via in-depth study. Thus, the intervention study was carried out here to explore MRSA transmission and infection trend in NICUs. Patients recruited in this study were from the NICU of a university-affiliated hospital with more than 2000 beds in Xiamen, China. We analyzed the compliance of multifaceted interventions, the clinical characteristics, and the MRSA infection trend. This study aimed to investigate the association of bundle interventions with the MRSA transmission and to provide effective infection control measures to prevent MRSA transmission in NICUs.

\section{Materials and Methods}

2.1. Setting and Design. The First Affiliated Hospital of Xiamen University is a Joint Commission International (JCI) accredited academic medical center hospital which acquired certification in 2015 and HIMSS EMRAM level 7 in 2017. It houses an 80 -bed, Level III NICU. The NICU has 3 private rooms and 4 open bays. 11,277 neonates were admitted to the NICU between January 1, 2014, and December 31, 2017. The relationship between MRSA infection or colonization and these patients was analyzed based on those neonates. During this period, 5,305 neonates were retrospectively reviewed. A bundle intervention study was conducted covering active surveillance, isolation precautions, and hand hygiene promotion, and 5,972 neonates were hospitalized from January 1, 2016, to December 31, 2017.
The collected data, including the medical records of the cases, were extracted from the HAI real-time monitoring system connected with the electronic medical record and inspection system. Medical records were reviewed: this included the demographics, clinical care, microbiologic data, antibiotic resistance, isolation order, and outcomes of the patients with infection or colonization MRSA.

2.2. Surveillance and Cultures. Following the internal University Medicine Goettingen (UMG) guidelines, neonates having high risk factors of MRSA carriers were contacted in isolation, placed in a private room, and then screened for MRSA on admission. The neonate's mother then acts as a potential carrier of the infectious disease and has transferred the infection between hospitals and becomes the high-risk factor for the development of MRSA. Samples of nasal secretions were obtained with a swab for MRSA screened within 24 hours after their admission to the NICU. Bacterial isolation and antimicrobial susceptibility testing were performed in accordance with the methodology of the Clinical and Laboratory Standards Institute [29]. Using the disk diffusion method with S aureus ATCC 25923 as the quality control strain, susceptibility testing was routinely performed. The isolated Staphylococcus aureus was cultured with oxacillin at $2 \mu \mathrm{g} / \mathrm{ml}$. If Staphylococcus aureus could grow in oxacillin at a concentration greater than $2 \mu \mathrm{g} / \mathrm{ml}$, it was determined to be MRSA.

WHO's (World Health Organization's) "Five Moments for Hand Hygiene" was adopted to assess healthcare workers hand hygiene compliance. Two infection control nurses evaluated hand hygiene compliance though direct observation. The observers remained unchanged during the study. In addition, the contact isolation compliance with MRSA infection or colonization neonates were monitored by infection control nurses. HAIs were verified in accordance with the National Healthcare Safety Network's (NHSN's) surveillance definitions by the Centers for Disease Control and Prevention $[30]$.

2.3. Bundle Interventions. Newborns whose mothers were diagnosed with infection and those transferred from other hospitals were the high-risk factors for the development of MRSA carriers. Accordingly, they would be screened for MRSA. They were placed on contact precautions in a private room following standard precautions until pathogen culture results were reported.

To identify the colonized or infected neonates with MRSA, the doctors in charge should prescribe contact isolation to start the bundle interventions. There are many ways to isolate patients via nurses (e.g., pathogen isolation, alcohol disinfection of the bed, hanging blue contact isolation mark on the door and bed of isolation ward, "MRSA" sign on the patient's electronic medical record, and yellow button on the wristband). Only after all the measures are completed would it be considered a successful intervention. Fractions were used to calculate the intervention success rate. The denominator is the number of patients who should be isolated, and the numerator is the number of patients receiving successful intervention. The medical supplies of 
TABLE 1: Comparison of incidence and resistance rate of MRSA.

\begin{tabular}{cccccc}
\hline Year & Admissions & Neonates with MRSA isolates & Incidence of MRSA \%o & Staphylococcus aureus & Resistance rate of MRSA \% \\
\hline 2014 & 2826 & 16 & 5.66 & 68 & 23.53 \\
2015 & 2479 & 18 & 7.26 & 66 & 27.27 \\
2016 & 2872 & 22 & 7.66 & 69 & 31.88 \\
2017 & 3100 & 20 & 6.45 & 58 & 34.48 \\
\hline
\end{tabular}

each neonate with MRSA were used solely for that patient (e.g., stethoscope). The bed unit was wiped by nurses with disinfectant three times a day. HCWs (healthcare workers) were trained on proper prevention and how to best control MRSA transmission in a hospital setting, and hand hygiene activities were promoted to improve the hand hygiene compliance of HCWs.

The compliance of contact isolation was supervised by infection control nurses and the implementation was supervised by the infection control professional weekly. The bundle interventions also constructed a supervision mechanism for the medical staff. An official automatic (OA) network would record and report monthly incidents of misconduct where doctors did not prescribe contact isolation advice or nurses did not implement contact isolation. These misconducts were calculated into performance appraisal.

Major monitoring indicators covered the following:

(i) MRSA detection rate of Staphylococcus aureus

(ii) The incidence of events of colonization or infection with MRSA neonates

(iii) Rates of healthcare-associated MRSA infections per 1, 000 inpatients in NICU

(iv) Rates of community-acquired MRSA infections per 1, 000 inpatients in NICU

(v) The hand hygiene compliance of healthcare workers

2.4. Statistical Analysis. In this study, the statistical analyses were performed using SPSS 13.0 (SPSS Inc., Chicago, IL, USA). The continuous variables were presented as mean \pm standard deviation (mean \pm SD), and the categorical variables were expressed as number and percentage (\%). Continuous variables were analyzed using Student's $t$-test and categorical variables were analyzed using the $\chi^{2}$ test. Pearson's correlation analysis was performed to investigate the relationship between the rate of HA-MRSA and the rate of contact isolation, HAI, and hand hygiene. All $P$ values were 2 tailed, and $P<0.05$ was considered as significantly different.

\section{Results}

3.1. Clinical Characteristics of MRSA Colonized or Infected Neonates. During the study period, 11,277 patients were admitted to the NICU. Among all patients, males made up $54.90 \%$, females made up $45.10 \%$, and 3,383 (30.00\%) were either transferred from another hospital or admitted from home. The median length of stay in the NICU was $9.65 \pm$ 10.63 days (range 1-97). 261 neonates $(2.31 \%)$ had a culture grow Staphylococcus aureus and the median age was $15.73 \pm$ 8.05 days. The detection rate of MRSA was $29.12 \%(76 / 261)$ among total isolates of Staphylococcus aureus. The median length of stay in the NICU of the neonates with methicillinsusceptible $S$. aureus (MSSA) reached $8.68 \pm 8.08$ days. MRSA was $14.26 \pm 17.91$ days. The median length of MRSA was significantly longer than MSSA $(t=2.83, \mathrm{P}=0.005)$. MRSA infections covered 49 lower respiratory infections $(64.47 \%)$, 16 skin and soft tissue infections $(21.05 \%), 7$ gastrointestinal infections (9.21\%), 3 bacteremia, and 1 ventilator-associated pneumonia (VAP). Two of the 76 infected neonates $(2.63 \%)$ died but only one was due to an MRSA infection. From the periods of 2014-2015 and of 2016-2017, incidents of MRSA in NICUs increased from $6.41 \%$ o (34/5305) to $7.03 \%$ o (42/5972); there was no significant difference $\left(\chi^{2}=0.16, P=0.686\right)$. The methicillin resistance rate of Staphylococcus aureus rose from $25.37 \%(34 / 134)$ to $33.07 \%(42 / 127)$ after implementing bundle interventions; it was not significantly lower $\left(\chi^{2}=2.68\right.$, $P=0.102)$, as shown in Table 1 .

3.2. The Compliance of MRSA Contact Isolation. After implementing bundle interventions the compliance of MRSA contact isolation grew from $55.88 \%$ to $92.86 \%$ which was significantly higher than before $\left(\chi^{2}=14.21, \mathrm{P}=0.001\right)$, as shown in Table 2.

3.3. The Compliance of Hand Hygiene in NICUs. The compliance of hand hygiene before intervention from 2014 to 2015 reached $90.07 \%$ (363/403) and, after intervention from 2016 to 2017 , the compliance of hand hygiene rose to $93.23 \%$ (909/975). It was significantly higher $\left(\chi^{2}=4.00, P=0.045\right)$, as shown in Table 3.

3.4. HAI and MRSA Infection of Neonates. The rate of HAI in NICUs dropped from $1.87 \%$ to $1.71 \%$ after intervention; there was no significant difference $\left(\chi^{2}=0.41, \mathrm{P}=0.524\right)$. Median length of stay of the neonates with hospital-acquired MRSA after admission was $11.25 \pm 6.49$ days (range $4-21$ ). The rate of hospital-acquired MRSA after intervention decreased from $2.63 \%$ o to $1.00 \%$ o. It was significantly lower $\left(\chi^{2}=4.24\right.$, $\mathrm{P}=0.04$ ). The rate of community-acquired (CA) MRSA increased from $3.77 \%$ o to $6.03 \%$, therefore displaying no significant difference $\left(\chi^{2}=2.90, \mathrm{P}=0.089\right)$, as shown in Table 4 .

3.5. Isolated Strains of HAI. Among the 201 isolated strains, the main strains included $46 \mathrm{~K}$. pneumoniae, 35 P. aeruginosa, and $28 \mathrm{~A}$. baumannii. The most frequently isolated specimens were sputum $(45.27 \%)$, blood $(22.88 \%)$, urine $(8.46 \%)$, pus $(3.98 \%)$, etc. The isolated specimen from pus declined from 
TABLE 2: The compliance of MRSA contact isolation.

\begin{tabular}{|c|c|c|c|c|c|c|}
\hline Year & MRSA Neonates & $\begin{array}{l}\text { Neonates in a } \\
\text { private room }\end{array}$ & $\begin{array}{c}\text { Ordered contact } \\
\text { isolation }\end{array}$ & $\begin{array}{l}\text { Marked as } \\
\text { isolation }\end{array}$ & $\begin{array}{l}\text { Number in } \\
\text { compliance }\end{array}$ & $\begin{array}{c}\text { Rate of } \\
\text { compliance } \%\end{array}$ \\
\hline 2014 & 16 & 10 & 12 & 12 & 10 & 62.50 \\
\hline 2015 & 18 & 9 & 11 & 13 & 9 & 50.00 \\
\hline 2016 & 22 & 20 & 22 & 22 & 20 & 90.91 \\
\hline 2017 & 20 & 19 & 20 & 19 & 19 & 95.00 \\
\hline
\end{tabular}

TABLE 3: Compliance of hand hygiene in NICUs.

\begin{tabular}{lccc}
\hline Year & Moments of hand hygiene & Hand hygiene practices & Compliance \% \\
\hline 2014 & 189 & 167 & $88.35 \%$ \\
2015 & 214 & 196 & $91.59 \%$ \\
2016 & 346 & 318 & $91.91 \%$ \\
2017 & 629 & 591 & $93.96 \%$ \\
\hline
\end{tabular}

TABLE 4: HAI and MRSA infection in NICUs.

\begin{tabular}{|c|c|c|c|c|c|c|c|}
\hline \multirow{3}{*}{ Year } & \multirow{3}{*}{ Admissions } & \multirow{3}{*}{ Cases of HAI } & \multirow{3}{*}{ Rate of HAI\% } & \multicolumn{4}{|c|}{ MRSA } \\
\hline & & & & \multicolumn{2}{|c|}{$\mathrm{HA}$} & \multicolumn{2}{|c|}{ CA } \\
\hline & & & & Cases & Rate\%o & Cases & Rate\%o \\
\hline 2014 & 2826 & 50 & 1.77 & 6 & 2.12 & 10 & 3.54 \\
\hline 2015 & 2479 & 49 & 1.98 & 8 & 3.23 & 10 & 4.03 \\
\hline 2016 & 2872 & 39 & 1.36 & 4 & 1.39 & 18 & 6.26 \\
\hline 2017 & 3100 & 63 & 2.03 & 2 & 0.64 & 18 & 5.81 \\
\hline
\end{tabular}

$5.05 \%$ to $2.94 \%$ after the intervention, and no difference existed in the sequence of main infection sites.

3.6. Correlation between the Rate of Hospital-Acquired MRSA and Contact Isolation Compliance, HAI, and Hand Hygiene Compliance. To find the relationship between the rate of hospital-acquired MRSA, contact isolation compliance, HAI, and hand hygiene compliance, a total of 48 months of data (quarter of a year) were studied. The rate of hospital-acquired MRSA was significantly correlated with contact isolation compliance $(r=-0.888, \mathrm{P}<0.01)$, and the rate of hospitalacquired MRSA was not significantly correlated with that of HAI $(r=0.172, \mathrm{P}=0.525)$ or hand hygiene compliance $(\mathrm{r}=$ $-0.311, \mathrm{P}=0.241)$.

\section{Discussion}

Some studies have reported that there is a decreasing incidence in MRSA among hospitalized adults in the United States in recent years [31]. Dantes et al. estimated a 54\% decline in invasive MRSA [20]. However, MRSA is a common etiological agent of a life-threatening infection in NICUs, with increasing in-hospital mortality rates and prolonging hospital length of stay [3]. In this study, length of stay showed statistically significant differences between MRSA colonized neonates and MSSA colonized neonates and the similar results are shown in the previous report by Geraci et al. [32].

Our study also showed that lower respiratory tract infections with MRSA made up $64.47 \%$ while skin and soft tissue infections made up $21.05 \%$. Some studies have reported that
MRSA was mostly isolated from blood and bronchoalveolar lavage [3, 33]. Li et al. reported pneumonia (69, 53.1\%) was the most common infection of CA-MRSA in Chinese neonates [34]. Of 11,277 neonates, 76 (6.74\%o) had a culture grow MRSA including 20 neonates with NICU-acquired MRSA. This was lower than that of other studies which was, respectively, reported as $2 \%$ and $5.8 \%[12,35]$.

The annual incidence density of acquisition of MRSA ranged from 5.66 cases to 7.66 cases per 1000 admissions. This was lower than the $6.99 \%$ reported by Shirai et al. [3]. Geraci et al. reported that the acquisition of MRSA ranged from a maximum of 20.2 cases for 1000 patient-days to a minimum of 8.8 cases [32]. Infection surveillance is managed by the realtime infection monitor system in hospitals since 2009. The underreporting potential nosocomial infections and MRSA infection were not found in the ward during the study. MRSA infection was lower with the contributing factor being the characteristic difference between western populations and Chinese. The methicillin resistance rate of Staphylococcus aureus ranged from $23.53 \%$ to $34.48 \%$. HA-MRSA were more likely to develop into severe invasive infections. These infections could even cause death. In this study, one death was attributed to HA- MRSA infection. Harik et al. reported that $68 \%$ of MRSA cases were hospital-associated (HA) MRSA [36].

There was a relative risk of 24.2 for colonized patients in NICU to develop an MRSA infection during hospitalization [35]. MRSA contamination from high-touch surfaces is worrisome in developing countries [37]. A bundle of interventions are required in order for NICUs 
to prevent MRSA transmission. Single-room isolation is strongly recommended for neonates with high risk factors of MRSA.

Compliance with hand hygiene, active surveillance, and contact isolation either singularly or in conjunction have been insufficient and controversial. One study showed the decrease in MRSA acquisition was primarily attributed to the barrier effects of gowns and gloves followed by improved hand hygiene and lower HCW-patient contact rates [38]. However, other research reported only before and after patient contact rose from $40 \%$ to $76 \%$ for hand hygiene compliance. HAI and MRSA rates remained high and stable and there was no correlation between compliance and MRSA $[25,39]$.

A program of universal surveillance, contact precautions, hand hygiene, and institutional culture change was associated with the decrease in healthcare-associated transmissions of and infections with MRSA in an extensive healthcare system [21]. In our study, while the rates of healthcareassociated MRSA infections in NICUs had not changed during the two years before the intervention, they declined under the implementation of the bundle from 2.45 infections per 1000 admissions to 1.17 per 1000 admissions. During the same period, healthcare-associated infections declined slightly from $1.87 \%$ to $1.71 \%$. This was because the main infection sites of healthcare-associated infections (including respiratory tract, bacteremia, and urinary tract) and the main pathogens (including Klebsiella pneumoniae, Acinetobacter baumannii, and Pseudomonas aeruginosa) are Gram-negative conditional pathogens that adapt to the surface of a moist environment and are easy to cause respiratory infection in patients with low immunity. MRSA is a Gram-positive bacterium which makes it easy to colonize on the surface of the human body and spreads through hand contact which is the primary cause of SSTIs.

Accompanied by the implementation of bundle interventions, both hand hygiene compliance and contact isolation grew significantly. Hand hygiene compliance was improved from $90.07 \%$ to $93.23 \%$ and MRSA contact isolation increased from $55.88 \%$ to $92.86 \%$. The major cause of noncompliance was the lack of single room to isolate patients. By analyzing the correlations between the rate of hospital-acquired MRSA, contact isolation compliance, HAI, and hand hygiene compliance, we found that the rate of hospital-acquired MRSA was significantly correlated with that of contact isolation compliance. There was no significant correlation between the rate of hospital-acquired MRSA, HAI, and hand hygiene compliance. This revealed that screening for MRSA in addition to isolation precautions in MRSA bundle interventions are important and effective measures.

\section{Conclusions}

MRSA bundle interventions are capable of reducing healthcare-associated MRSA infections in NICUs. All research findings show that the bundle interventions are highly effective tools in MRSA prevention and should be adhered to routinely in order to improve contact isolation compliance.

\section{Data Availability}

The data used to support the findings of this study are included within the article.

\section{Ethical Approval}

This study was approved by the Ethics Committee of the First Affiliated Hospital of Xiamen University.

\section{Disclosure}

The funding entities have not influenced the study design, data collection and analysis, or preparation of the manuscript.

\section{Conflicts of Interest}

The authors declare that they have no conflicts of interest.

\section{Acknowledgments}

This study was financed by grants from the Science and Technology Project of the National Natural Science Foundation of China (No. 81601284) and the Natural Science Foundation of Fujian Province (2016J01640 to Jing Ran). We would also like to thank everyone who helped with this study.

\section{References}

[1] G. Messina, E. Ceriale, D. Lenzi, S. Burgassi, E. Azzolini, and P. Manzi, "Environmental contaminants in hospital settings and progress in disinfecting techniques," BioMed Research International, vol. 2013, Article ID 429780, 8 pages, 2013.

[2] A. Balkhair, Y. M. Al-Farsi, Z. Al-Muharrmi et al., "Epidemiology of multi-drug resistant organisms in a teaching hospital in oman: a one-year hospital-based study," The Scientific World Journal, vol. 2014, Article ID 157102, 6 pages, 2014.

[3] Y. Shirai, H. Arai, K. Tamaki et al., "Neonatal methicillinresistant Staphylococcus aureus colonization and infection," Journal of Neonatal-Perinatal Medicine, vol. 10, no. 4, pp. 439444, 2017.

[4] E. H. Ristagno, K. A. Bryant, L. F. Boland et al., "Effect of intranasal mupirocin prophylaxis on methicillin-resistant staphylococcus aureus transmission and invasive staphylococcal infections in a neonatal intensive care unit," Infection Control \& Hospital Epidemiology, vol. 39, no. 6, pp. 741-745, 2018.

[5] A. Semple, E. O’Curráin, D. O’Donovan, U. Ní Riain, and E. Moylett, "Neonatal methicillin-resistant Staphylococcus aureus surveillance and management practice: results of a National Irish Survey," Infection Control \& Hospital Epidemiology, vol. 35, no. 11, pp. 1438-1439, 2014.

[6] A. Dramowski, A. Madide, and A. Bekker, "Neonatal nosocomial bloodstream infections at a referral hospital in a middleincome country: burden, pathogens, antimicrobial resistance and mortality," Paediatrics and International Child Health, vol. 35, no. 3, pp. 265-272, 2015.

[7] R. C. Couto, E. A. A. Carvalho, T. M. G. Pedrosa, Ê. R. Pedroso, M. C. Neto, and F. M. Biscione, "A 10-year prospective surveillance of nosocomial infections in neonatal intensive care 
units," American Journal of Infection Control, vol. 35, no. 3, pp. 183-189, 2007.

[8] J. E. Ericson, V. O. Popoola, P. B. Smith et al., "Burden of invasive staphylococcus aureus infections in hospitalized infants," JAMA Pediatrics, vol. 169, no. 12, pp. 1105-1111, 2015.

[9] G. A. Filice, J. A. Nyman, C. Lexau et al., "Excess costs and utilization associated with methicillin resistance for patients with Staphylococcus aureus infection," Infection Control \& Hospital Epidemiology, vol. 31, no. 4, pp. 365-373, 2010.

[10] Y. C. Kim, M. H. Kim, J. E. Song et al., "Trend of methicillinresistant Staphylococcus aureus (MRSA) bacteremia in an institution with a high rate of MRSA after the reinforcement of antibiotic stewardship and hand hygiene," American Journal of Infection Control, vol. 41, no. 5, pp. e39-e43, 2013.

[11] O. Dolapo, R. Dhanireddy, and A. J. Talati, "Trends of Staphylococcus aureus bloodstream infections in a neonatal intensive care unit from 2000-2009," BMC Pediatrics, vol. 14, no. 1, article 121, 2014.

[12] V. O. Popoola, A. Budd, S. M. Wittig et al., "Methicillin-resistant Staphylococcus aureus transmission and infections in a neonatal intensive care unit despite active surveillance cultures and decolonization: Challenges for infection prevention," Infection Control and Hospital Epidemiology, vol. 35, no. 4, pp. 412-418, 2014.

[13] R. W. Loftus, F. Dexter, and A. D. Robinson, "Methicillinresistant Staphylococcus aureus has greater risk of transmission in the operating room than methicillin-sensitive S aureus," American Journal of Infection Control, vol. 46, no. 5, pp. 520525, 2018.

[14] R. Loftus, F. Dexter, A. Robinson, and A. Horswill, "Desiccation tolerance is associated with Staphylococcus aureus hypertransmissibility, resistance and infection development in the operating room," Journal of Hospital Infection, vol. 100, no. 3, pp. 299-308, 2018.

[15] M. Inoue, K. Uchida, T. Ichikawa et al., "Contaminated or dirty wound operations and methicillin-resistant Staphylococcus aureus (MRSA) colonization during hospitalization may be risk factors for surgical site infection in neonatal surgical patients," Pediatric Surgery International, vol. 34, no. 11, pp. 1209-1214, 2018.

[16] S. Eldirdiri, J. Lee, A. Jack, A. Wright, A. Findlay, and G. Phillips, "Outbreak of gentamicin-resistant, meticillin-susceptible Staphlococcus aureus on a neonatal unit," Journal of Hospital Infection, vol. 98, no. 4, pp. 419-424, 2018.

[17] R. Pierce, J. Lessler, V. Popoola, and A. Milstone, "Meticillinresistant Staphylococcus aureus (MRSA) acquisition risk in an endemic neonatal intensive care unit with an active surveillance culture and decolonization programme," Journal of Hospital Infection, vol. 95, no. 1, pp. 91-97, 2017.

[18] E. Couvé-Deacon, F. Mons, F. Garnier et al., "Neonatal outbreak of methicillin-resistant staphylococcus aureus clone geraldine: a bundle of measures to halt transmission," Infection Control and Hospital Epidemiology, vol. 38, no. 6, pp. 749-751, 2017.

[19] K. Williams, S. Hopkins, D. Turbitt et al., "Survey of neonatal unit outbreaks in North London: identifying causes and risk factors," Journal of Hospital Infection, vol. 88, no. 3, pp. 149-155, 2014.

[20] R. Dantes, Y. Mu, R. Belflower et al., "National burden of invasive methicillin-resistant Staphylococcus aureus infections, United States, 2011," JAMA Internal Medicine, vol. 173, no. 21, pp. 1970-1979, 2013.
[21] R. Jain, S. M. Kralovic, M. E. Evans et al., "Veterans affairs initiative to prevent methicillin-resistant Staphylococcus aureus infections," The New England Journal of Medicine, vol. 364, no. 15, pp. 1419-1430, 2011.

[22] M. Jones, J. Ying, B. Huttner et al., "Relationships between the importation, transmission, and nosocomial infections of methicillin-resistant staphylococcus aureus: an observational study of 112 veterans affairs medical centers," Clinical Infectious Diseases, vol. 58, no. 1, pp. 32-39, 2013.

[23] R. Kullar, A. Vassallo, S. Turkel, T. Chopra, K. S. Kaye, and S. Dhar, "Degowning the controversies of contact precautions for methicillin-resistant Staphylococcus aureus: A review," American Journal of Infection Control, vol. 44, no. 1, pp. 97-103, 2016.

[24] S. A. Salati and A. Al Kadi, "Hand hygiene practices in medical students: a follow-up study," International Scholarly Research Notices, vol. 2014, Article ID 591879, 5 pages, 2014.

[25] L. F. Borges, L. A. Rocha, M. J. Nunes, and P. P. Gontijo Filho, "Low compliance to handwashing program and high nosocomial infection in a Brazilian Hospital," Interdisciplinary Perspectives on Infectious Diseases, vol. 2012, Article ID 579681, 5 pages, 2012.

[26] R. E. Nelson, V. W. Stevens, K. Khader et al., "Economic analysis of veterans affairs initiative to prevent methicillinresistant staphylococcus aureus infections," American Journal of Preventive Medicine, vol. 50, no. 5, pp. S58-S65, 2016.

[27] B. Y. Lee, R. R. Bailey, K. J. Smith et al., "Universal methicillinresistant Staphylococcus aureus (MRSA) surveillance for adults at hospital admission: an economic model and analysis," Infection Control \& Hospital Epidemiology, vol. 31, no. 6, pp. 598-606, 2010.

[28] W. Chaboyer, T. Bucknall, J. Webster et al., "The effect of a patient centred care bundle intervention on pressure ulcer incidence (INTACT): a cluster randomised trial," International Journal of Nursing Studies, vol. 64, pp. 63-71, 2016.

[29] R. L. Sinkowitz-Cochran, K. H. Burkitt, T. Cuerdon et al., "The associations between organizational culture and knowledge, attitudes, and practices in a multicenter veterans affairs quality improvement initiative to prevent methicillin-resistant Staphylococcus aureus," American Journal of Infection Control, vol. 40, no. 2, pp. 138-143, 2012.

[30] Institute CaLS, "Methods for dilution antimicrobial susceptiblity tests for bacteria that grow aerobically; approved standardeighth edition," Clinical and Laboratory Standards Institute, vol. 29, no. 11, 2008.

[31] T. C. Horan, M. Andrus, and M. A. Dudeck, "CDC/NHSN surveillance definition of health care-associated infection and criteria for specific types of infections in the acute care setting," American Journal of Infection Control, vol. 36, no. 5, pp. 309-332, 2008.

[32] D. M. Jacobs and A. Shaver, "Prevalence of and outcomes from Staphylococcus aureus pneumonia among hospitalized patients in the United States, 2009-2012," American Journal of Infection Control, vol. 45, no. 4, pp. 404-409, 2017.

[33] D. M. Geraci, M. Giuffrè, C. Bonura et al., "Methicillin-resistant Staphylococcus aureus colonization: a three-year prospective study in a neonatal intensive care unit in Italy," PLoS ONE, vol. 9, no. 2, Article ID e87760, 2014.

[34] M. Abdel-Maksoud, M. El-Shokry, G. Ismail et al., "Methicillinresistant Staphylococcus aureus recovered from healthcareand community-associated infections in Egypt," International Journal of Bacteriology, vol. 2016, Article ID 5751785, 5 pages, 2016. 
[35] S. Li, X. Ning, W. Song et al., " Clinical and molecular characteristics of community-acquired methicillin-resistant Staphylococcus aureus infections in Chinese neonates," APMIS-Acta Pathologica, Microbiologica et Immunologica Scandinavica, vol. 123, no. 1, pp. 28-36, 2015.

[36] F. N. Zervou, I. M. Zacharioudakis, P. D. Ziakas, and E. Mylonakis, "MRSA colonization and risk of infection in the neonatal and pediatric ICU: a meta-analysis," Pediatrics, vol. 133, no. 4, pp. e1015-e1023, 2014.

[37] N. S. Harik, G. Com, X. Tang, M. Melguizo Castro, M. E. Stemper, and J. L. Carroll, "Clinical characteristics and epidemiology of methicillin-resistant Staphylococcus aureus (MRSA) in children with cystic fibrosis from a center with a high MRSA prevalence," American Journal of Infection Control, vol. 44, no. 4, pp. 409-415, 2016.

[38] D. Lin, Q. Ou, J. Lin, Y. Peng, and Z. Yao, "A meta-analysis of the rates of Staphylococcus aureus and methicillin-resistant $S$ aureus contamination on the surfaces of environmental objects that health care workers frequently touch," American Journal of Infection Control, vol. 45, no. 4, pp. 421-429, 2017.

[39] A. Harris, D. Morgan, L. Pineles, E. Perencevich, and S. Barnes, "Deconstructing the relative benefits of a universal glove and gown intervention on MRSA acquisition," Journal of Hospital Infection, vol. 96, no. 1, pp. 49-53, 2017. 


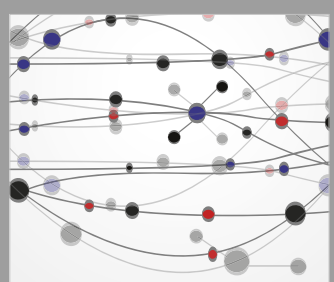

The Scientific World Journal
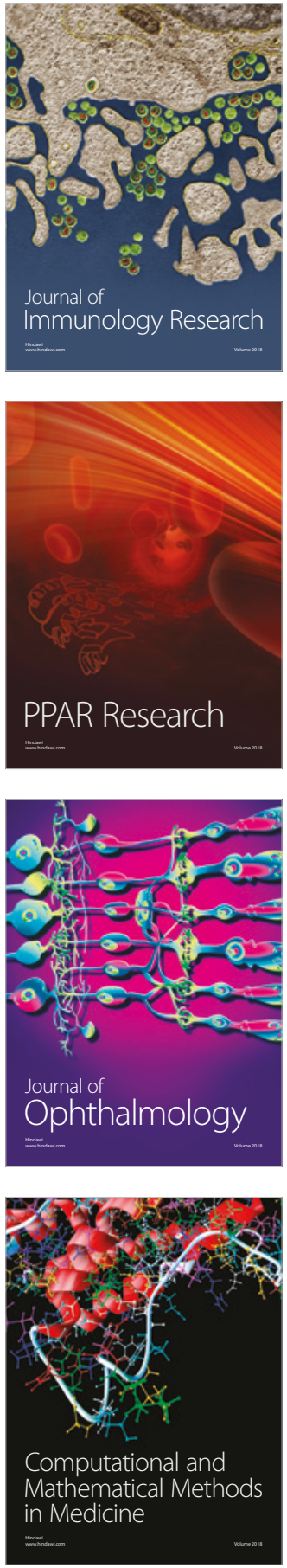

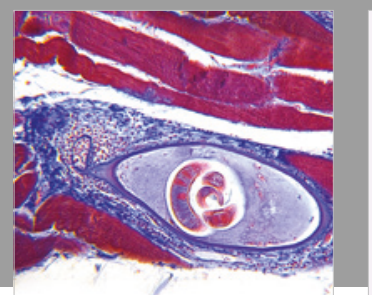

Gastroenterology Research and Practice

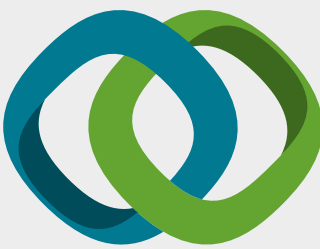

\section{Hindawi}

Submit your manuscripts at

www.hindawi.com
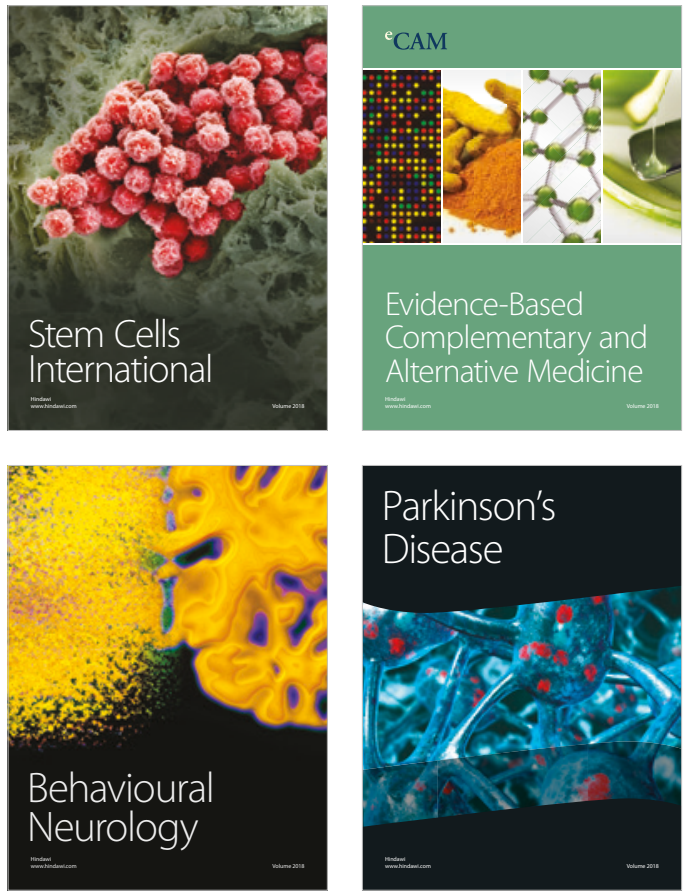

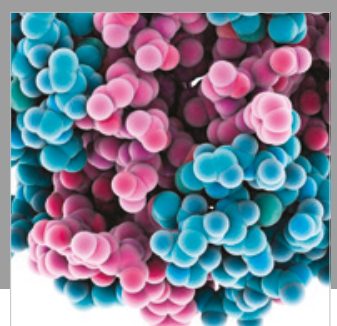

ournal of

Diabetes Research

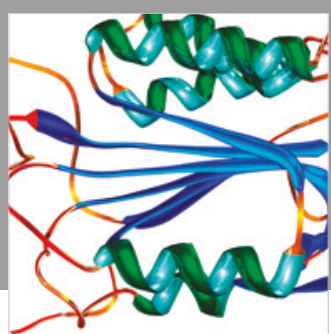

Disease Markers
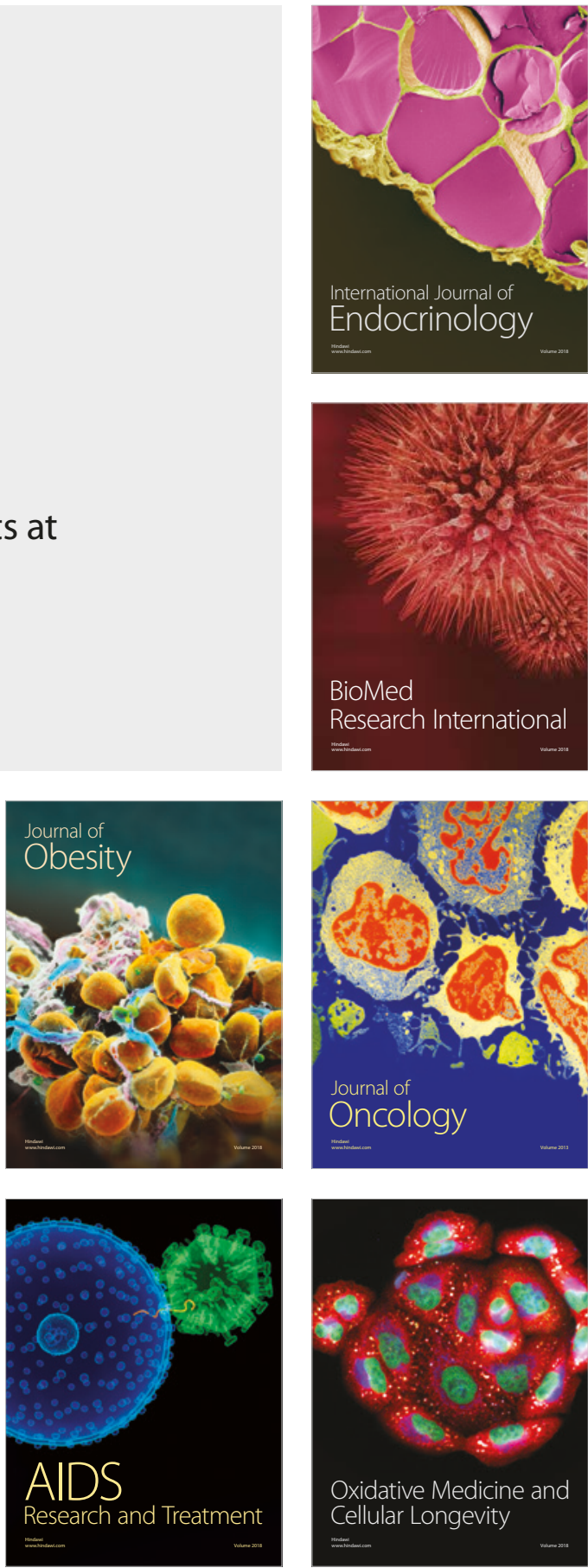